\title{
Combinatorial structures associated with low dimensional second class of non-Lie filiform Leibniz algebra
}

\begin{abstract}
In this talk we propose a graphical representation of some classes of Leibniz algebras. With each of algebra from these classes we associate a graph. This assignment enables us to reformulate some structural properties of the Leibniz algebras in terms of some conditions for the graphs. The talk focuses on a so-called filiform Leibniz algebras. It is well-known that this class is split into three subclasses called first, second and third class denoted, in dimension $n$ over a field $\mathrm{K}$, by $\operatorname{FLbn}(\mathrm{K}), \operatorname{SLbn}(\mathrm{K})$ and $\mathrm{TLbn}(\mathrm{K})$, respectively. In this article, we concern more on the combinatorial structures associated with $\operatorname{SLbn}(K)$ in lowdimensions.
\end{abstract}

Keyword: Leibniz algebras; Filiform Leibniz algebras; Graph theory 